\title{
Saprophagous insect larvae, Drosophila melanogaster, profit from increased species richness in beneficial microbes
}

\author{
M. Rohlfs \& L. Kürschner \\ Zoological Institute, Department of Evolutionary Ecology and Genetics, Christian-Albrechts-University of Kiel, Germany
}

\section{Keywords}

Aspergillus nidulans, competition, insect-

fungus interactions, species richness, yeast

\section{Correspondence}

Marko Rohlfs (corresponding author), Zoological Institute, Department of Evolutionary Ecology and Genetics, ChristianAlbrechts-University of Kiel, Germany.

E-mail: rohlfs@zoologie.uni-kiel.de

Received: August 5, 2009; accepted: September 16, 2009.

doi: 10.1111/j.1439-0418.2009.01458.x

\begin{abstract}
Female fruit flies, Drosophila melanogaster, lay their eggs on decaying plant material. Foraging fly larvae strongly depend on the availability of dietary microbes, such as yeasts, to reach the adult stage. In contrast, strong interference competition with filamentous fungi can cause high mortality among Drosophila larvae. Given that many insects are known for employing beneficial microbes to combat antagonistic ones, we hypothesized that fly larvae engaged in competition with the noxious mould Aspergillus nidulans benefit from the presence of dietary yeast species, especially when they are associated with increasingly species rich yeast communities (ranging from one to six yeast species per community). On a nutrient-limited fruit substrate infested with A. nidulans, both larval survival and development time were positively affected by more diverse yeast communities. On a mould-free fruit substrate, merely larval development but not survival was found to be affected by increasing species richness of dietary yeasts. Not only yeast diversity had an effect on D. melanogaster life-history traits, but also the identity of the yeast combinations. These findings demonstrate the importance of the structure and diversity of microbial communities in mutualistic animalmicrobe interactions.
\end{abstract}

\section{Introduction}

To make food resources accessible, provide supplemental nutrition and fend off parasites, animals, including humans, often rely on symbiotic microorganisms (Dethlefsen et al. 2007; Rosenberg et al. 2007; Klepzig et al. 2009). In contrast, many microbes, such as filamentous fungi or bacteria, render food sources unpalatable to animals (Janzen 1977; Burkepile et al. 2006; Rozen et al. 2008). Insects often face diverse antagonistic interactions with filamentous fungi, this could be one reason why they may have been selected for establishing associations with specific bacteria or yeasts that impair fungal growth, possibly on grounds of their capacity to synthesize anti-fungal metabolites (Kaltenpoth et al. 2005; Cardoza et al. 2006; Little et al. 2006; Scott et al. 2008; Haeder et al. 2009; Lam et al. 2009; Rodrigues et al. 2009).

Drosophilid flies are a prime example of the large group of saprophagous insects whose larvae develop on dead organic material, e.g. rotting plant tissue (Shorrocks 1982), that is concomitantly inhabited by various microorganisms. For a successful development the fly larvae depend on the availability of dietary yeasts (Begon 1982; Anagnostou et al. 2009) that may be transferred to the breeding site by adult flies (Wertheim et al. 2002; Rohlfs and Hoffmeister 2005), suggesting a mutualistic Drosophila-yeast relationship (Vega and Dowd 2005). Nevertheless, the immature insects may also encounter noxious microbes, e.g. mould fungi that can cause high mortality among the animals (Rohlfs et al. 2005). Recent pharmacological tests indicate that the interference 
competition between insects and fungi may be mediated by the secretion of mycotoxins (Rohlfs and Obmann 2009).

Here, we tested the hypothesis that, in addition to their role in Drosophila melanogaster nutrition, dietary yeasts enhance insect survival in the presence of a competing fungus, Aspergillus nidulans. Insect-associated yeasts have been shown to curtail growth of antagonistic fungi in other systems (Adams et al. 2008; Rodrigues et al. 2009), and might be capable of detoxifying mycotoxins (Dowd 1992). We predicted that different yeast species and communities may have different effects on insect development, either by inhibiting mould growth or by changing the quality of the diet. Especially, we hypothesized that increased yeast species richness may positively affect the larval development of $D$. melanogaster.

\section{Methods}

\section{Organisms}

The D. melanogaster population originated from isofemale lines caught in Kiel, Northern Germany, in 2003 and were cultured under standard condition (see Rohlfs et al. 2005). Larvae used in the experiment originated from eggs sterilized with 50\% sodium hypochlorite. The freshly hatched lst instar larvae could then be transferred with a fine brush under sterile conditions.

The yeasts chosen were Kluyveromyces lactis (DSM4909), Metschnikowia pulcherrima (DSM70321), Pichia toletana (DSM70390), Saccharomyces cerevisiae (DSM70449), Cryptococcus albidus (DSM70215) and Rhodotorula mucilaginosa (DSM70404), which were cultured on malt extract agar at $28^{\circ} \mathrm{C}$. After 4 days of cultivation, the yeast colonies were washed off with sterile Ringer solution. For the experiment, yeast suspensions comprising different combinations of yeast species were prepared. There were 63 possible yeast combinations. Independently of the yeast species richness and combination in each experimental treatment, the initial cell number was kept constant at 1000000 cells per $\mu$, i.e. with increasing species richness the number of cells per yeast species decreased accordingly. A mycotoxin producing wild type strain of Aspergillus nidulans (RDIT2.3) was cultured under the same conditions as the above mentioned yeast species. This strain has been shown to seriously increased Drosophila larval mortality due to competitive but non-pathogenic interactions (Rohlfs et al. 2009). After 4 days of cultivation, the mature conidiospores were washed off with sterile
Ringer solution. The desired number of 1000 conidia per $\mu$ l was obtained by using a haemocytometer.

\section{Experimental setup}

We used a fruit medium (banana) to simulate natural conditions at which the insect larvae depend on the availability of yeast to reach the adult stage. Finely crushed banana mixed with the same volume of tap water and $15 \mathrm{~g}$ agar per litre banana-water mixture. $1 \mathrm{ml}$ medium was pipetted into $2 \mathrm{ml}$ micro tubes and autoclaved. Without the addition of dietary microbes, this sterile fruit substrate does not support the development of Drosophila larvae whatsoever (Dorsch 2007). A. nidulans was given a developmental head-start of $60 \mathrm{~h}$ by transferring $\mathrm{l} \mu \mathrm{l}$ conidia suspension prior to the inoculation with the yeast cells. Ten larvae each were transferred into the tubes, the tubes were covered with sterile cotton plugs and incubated in a climate chamber at $25^{\circ} \mathrm{C}$ and a $16: 8 \mathrm{~h} \mathrm{~L}: \mathrm{D}$ cycle. For a randomly chosen subset (eight combinations) of the 3-spp. treatment, the full 1-spp. and the 6-spp. treatment we ran the same experiment at mould-free conditions. The development of larvae was followed until adult emergence. Since adult body weight in D. melanogaster has repeatedly been shown to decrease with increasing development time (Blanckenhorn 1999; Wertheim et al. 2002), we here report only on larval survival to the adult stage and development time (days from larval transfer to adult emergence). There were $n=20$ replicates per treatment.

\section{Statistical analysis}

We used ANOva models to test for the effects of yeast species richness and community composition on the survival of Drosophila larvae. Proportional insect survival data were square root-arcsine-transformed. Species combination was considered as a factor nested under species richness. The analyses of the nested ANOVA model were performed with sPSS 17.0.

\section{Results and Discussion}

Our experiment revealed positive effects of increasingly species-rich yeast communities on the development of $D$. melanogaster larvae that were forced to feed in the presence of the toxic mould A. nidulans: survival was significantly higher (ca. 20\%) (species number: $\mathrm{F}_{5,1197}=5.013, \mathrm{P}<0.001$; species combination: $\left.\mathrm{F}_{57,1197}=2.999, \mathrm{P}<0.001\right)$ and development time significantly shorter (ca. 0.75 days) (species 
number: $\mathrm{F}_{5,887}=2.438, \mathrm{P}=0.033$; species combination: $\left.\mathrm{F}_{57,887}=2.410, \quad \mathrm{P}<0.001\right)$ when microbial communities were richer in species (Fig. la, c). Subsequent regression analyses indicated that development time had a linear relationship with yeast species richness (Fig. 1c); however, insect survival was non-linearly related to yeast species richness and appeared not to reach saturation at very high species richness (Fig. la). Thus, D. melanogaster larvae may achieve an even higher developmental success at still more diverse yeast communities.

Various, mutually non-exclusive mechanisms might explain the observed positive influence of biodiversity on insect survival. (1) Yeasts may secrete anti-fungal compounds that curtail mould growth (e.g. Liu et al. 2007). Species-rich communities may produce various, functionally different defence metabolites that, when acting in combination, may be more effective in suppressing mould develop-
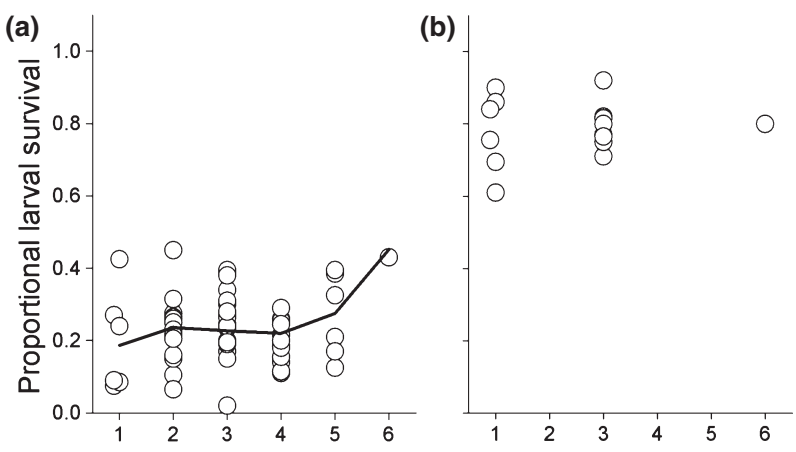

(c)

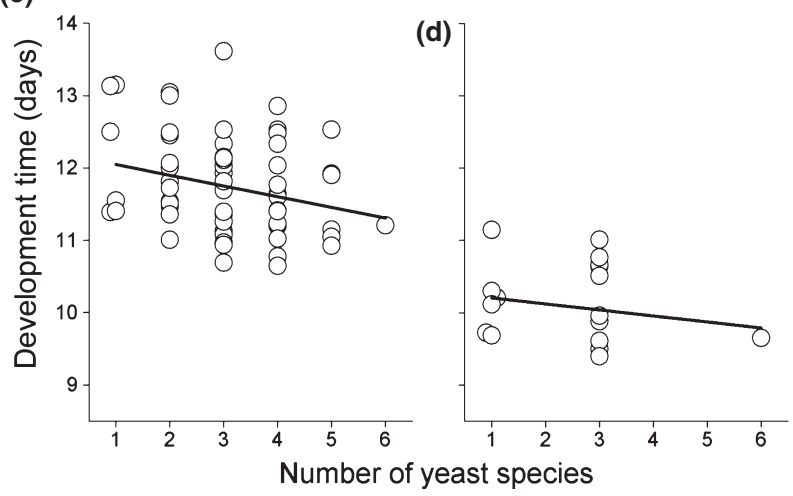

Fig. 1 Mean Drosophila melanogaster larval survival and development time in each yeast treatment as a function of yeast species richness under mouldy conditions (a and c) and under mould-free conditions ( $b$ and $d$ ). Regression equations: (a) $y=0.246 x-$ $0.089 x^{2}+0.010 x^{3}+0.020 ; R^{2}=0.013, P=0.001$, this is the most parsimonious regression model; (b) no effect of yeast species richness; $\quad$ (c) $\quad y=-0.148 x+12.195 ; \quad R^{2}=0.012, \quad P=0.002 ; \quad$ (d) $y=-0.083 x+10.288 ; R^{2}=0.029, P=0.010$. For clarity, the data of the one species treatment are staggered around the values on the $x$-axis. (see text for statistical details). ment. (2) Species-rich yeast communities may provide a functionally diverse means of detoxification in the fruit substrate and/or the insect gut (Dowd 1992). Yeast-borne degradation of mycotoxins might prevent the insect detoxification system (Li et al. 2007) from being overstrained and hence allow allocation of resources and energy to larval growth. (3) A diverse microbial diet might supply the insect larvae with essential and complementary nutrients (dietary mixing, Bernays et al. 1994) that enhance specific physiological processes (e.g. mycotoxin detoxification) supporting the insects in combating the fungal competitor. In absence of the fungal competitor, however, we found no beneficial effect of yeast species enrichment on insect survival (species number: $\mathrm{F}_{2,213}=0.048, \mathrm{P}=0.953$; species combination: $\mathrm{F}_{14,213}=1.915, \mathrm{P}<0.001$ ) (Fig. 1b). Yet, development time was slightly accelerated (ca. half a day) (species number: $\mathrm{F}_{2,213}=10.07, \mathrm{P}<$ 0.001 ; species combination: $\mathrm{F}_{14,213}=9.512, \quad \mathrm{P}<$ 0.001) (Fig. 1d), indicating subtle influences on insect performance, which might be of relevance when Drosophila develops on qualitatively different substrates (Starmer and Aberdeen 1990) or, as indicated by the present study, on those infested with noxious microbes.

In addition to the positive influence of yeast species richness on Drosophila development in the presence of the fungal competitor, there was a strong effect of yeast community composition (see statistical results above). Thus, the impact of single yeast species or specific yeast combinations mediates significant changes in insect developmental success (see low explanatory power of regression models in Fig. 1). Without detailed analyses of changes in the yeast communities, we cannot exclude that those yeast species providing optimal conditions already in the one-spp. treatment (see Fig. 1) outcompete all other yeasts, so that species combination is in fact more important than species richness for Drosophila development. On the other hand, association with more diverse microbial communities may increase the probability that these beneficial species are present. Independently of the mechanism underlying the observed phenomenon, yeasts may critically mitigate the selection pressure imposed on Drosophila populations by competing fungi and thereby reduce the evolutionary costs that accompany evolution of protection against the fungal competitor (Wölfle et al. 2009). Moreover, being associated with species-rich microbial communities may buffer against evolutionary changes in the antagonistic ones (Rosenberg et al. 2007). How drosophilids achieve association 
with beneficial microbial communities of varying diversity (Vega and Dowd 2005), however, is still an underexplored aspect of the natural ecology of this model insect.

Our analysis provides first evidence for a positive effect of dietary microbial biodiversity on saprophagous insects forced to develop in the presence of a competing toxic mould fungus. Though the underlying mechanisms of this effect remain to be investigated, our study highlights the importance of the structure and diversity of (possibly defensive) microbial communities in mutualistic animal-microbe interactions.

\section{Acknowledgements}

Nancy P. Keller is acknowledged for providing the Aspergillus strain used in this study. Two anonymous reviewers provided valuable comments. The work was partially funded by a DFG grant to M.R. (Ro2523/2-1).

\section{References}

Adams A, Six D, Adams S, Holben W, 2008. In vitro interactions between yeasts and bacteria and the fungal symbionts of the Mountain Pine Beetle (Dendroctonus ponderosae). Microb. Ecol. 56, 460-466.

Anagnostou C, LeGrand EA, Rohlfs M, 2009. Friendly food for fitter flies? - Influence of the microbial food on diet choice and parasitoid resistance in Drosophila. Oikos (in press).

Begon M 1982. Yeast and Drosophila. In: The genetics and biology of Drosophila. Ed. by Ashburner M, Carson HL, Thompson Jr. JN, Academic Press Inc., London, pp. 345-384.

Bernays EA, Bright KL, Gonzalez N, Angel J, 1994. Dietary mixing in a generalist herbivore: tests of two hypotheses. Ecology 75, 1997-2006.

Blanckenhorn WU, 1999. Different growth responses to temperature and resource limitation in three fly species with similar life histories. Evol. Ecol. 13, 395409.

Burkepile DE, Parker JD, Woodson CB, Mills HJ, Kubanek J, Sobecky PA, Hay ME, 2006. Chemically mediated competition between microbes and animals: microbes as consumers in food webs. Ecology 87, 2821-2831.

Cardoza YJ, Klepzig KD, Raffa KF, 2006. Bacteria in oral secretions of an endophytic insect inhibit antagonistic fungi. Ecol. Entomol. 31, 636-645.

Dethlefsen L, McFall-Ngai M, Relman DA, 2007. An ecological and evolutionary perspective on humanmicrobe mutualism and disease. Nature 449, 811-818.
Dorsch M, 2007. Einfluss der mikrobiellen Nahrung auf die Entwicklung von Drosophila. Diploma thesis, Christian-Albrechts-Univesity of Kiel, Kiel, Germany

Dowd PF, 1992. Insect fungal symbionts: a promising source of detoxifying enzymes. J. Indust. Microbiol. Biotechnol. 9, 149-161.

Haeder S, Wirth R, Herz H, Spiteller D, 2009. Candicidinproducing Streptomyces support leaf-cutting ants to protect their fungus garden against the pathogenic fungus Escovopsis. Proc. Natl Acad. Sci. USA 106, 4742-4746.

Janzen DH, 1977. Why fruits rot, seeds mold and meat spoils. Am. Nat. 111, 691-713.

Kaltenpoth M, Göttler W, Herzner G, Strohm E, 2005. Symbiotic bacteria protect wasp larvae from fungal infestation. Curr. Biol. 15, 475-479.

Klepzig KD, Adams AS, Handelsman J, Raffa KF, 2009. Symbioses: a key driver of insect physiological processes, ecological interactions, evolutionary diversification, and impacts on humans. Environ. Entomol. 38, 67-77.

Lam K, Thu K, Tsang M, Moore M, Gries G, 2009. Bacteria on housefly eggs, Musca domestica, suppress fungal growth in chicken manure through nutrient depletion or antifungal metabolites. Naturwissenschaften 96, 1127-1132.

Li X, Schuler MA, Berenbaum MR, 2007. Molecular mechanisms of metabolic resistance to synthetic and natural xenobiotics. Ann. Rev. Entomol. 52, 231-253.

Little AEF, Murakami T, Mueller UG, Currie CR, 2006. Defending against parasites: fungus-growing ants combine specialized behaviours and microbial symbionts to protect their fungus gardens. Biol. Lett. 2, 12-16.

Liu X, Wang J, Gou P, Mao C, Zhu Z-R, Li H, 2007. In vitro inhibition of postharvest pathogens of fruit and control of gray mold of strawberry and green mold of citrus by aureobasidin A. Inter. J. Food Microbiol. 119, 223-229.

Rodrigues A, Cable R, Mueller U, Bacci M, Pagnocca F 2009. Antagonistic interactions between garden yeasts and microfungal garden pathogens of leaf-cutting ants. Antonie van Leeuwenhoek doi:10.1007/s10482-0099350-7 (online first).

Rohlfs M, Hoffmeister TS, 2005. Maternal effects increase survival probability in Drosophila subobscura larvae. Entomol. Exp. Appl. 117, 51-58.

Rohlfs M, Obmann B, 2009. Species-specific responses of dew fly larvae to mycotoxins. Mycot. Res. 25, 103112.

Rohlfs M, Obmann B, Petersen R, 2005. Competition with filamentous fungi and its implications for a gregarious life-style in insects living on ephemeral resources. Ecol. Entomol. 30, 556-563.

Rohlfs M, Trienens M, Fohgrub U, Kempken F, 2009. Ecological and evolutionary interactions of mould and insects. In: The mycota XV physiology and genetics. 
Ed. by Anke T, Weber D, Springer-Verlag, Berlin, Heidelberg, pp. 131-151.

Rosenberg E, Koren O, Reshef L, Efrony R, Zilber-Rosenberg I, 2007. The role of microorganisms in coral health, disease and evolution. Nat. Rev. Micro. 5, 355-362.

Rozen DE, Engelmoer DJP, Smiseth PT, 2008. Antimicrobial strategies in burying beetles breeding on carrion. Proc. Natl Acad. Sci. USA 105, 17890-17895.

Scott JJ, Oh D-C, Yuceer MC, Klepzig KD, Clardy J, Currie CR, 2008. Bacterial protection of beetle-fungus mutualism. Science 322, 63.

Shorrocks B, 1982. The breeding sites of temperate woodland Drosophila. In: The genetics and biology of Drosophila 3b. Ed. by Ashburner M, Carson HL, Thompson Jr. JN, Academic Press, London, pp. 385-428.

Starmer WT, Aberdeen V, 1990. The nutritional importance of pure and mixed cultures of yeast in the development of D. mülleri larvae in Opuntia tissues and its relationship to host plant shifts. In: Ecological and evolutionary genetics of Drosophila. Ed. by Barker JSF, Starmer WT, MacIntyre RJ, Plenum Press,

New York, pp. 145-160.

Vega F, Dowd P, 2005. The role of yeasts as insect endosymbionts. In: Insect-fungal associations - ecology and evolution. Ed. by Vega $\mathrm{F}$ and Blackwell $\mathrm{M}$, Oxford University Press, New York.

Wertheim B, Marchais J, Vet LEM, Dicke M, 2002. Allee effect in larval resource exploitation in Drosophila: an interaction between density of adults, larvae and micro-organisms. Ecol. Entomol. 27, 608-617.

Wölfle S, Trienens M, Rohlfs M 2009. Experimental evolution of resistance against a competing fungus in Drosophila. Oecologia 161, 781-790. 